\title{
What is new in surgical treatment of vesicoureteric reflux?
}

\author{
Piet R. H. Callewaert
}

Received: 26 January 2007 / Accepted: 1 March 2007 / Published online: 20 March 2007

(C) Springer-Verlag 2007

\begin{abstract}
In addition to conventional open surgery and endoscopic techniques, laparoscopic correction of vesicoureteric reflux, sometimes even robot-assisted, is becoming an alternative surgical treatment modality for this condition in a number of centres around the world. At least for a subgroup of patients laparoscopists are trying to develop new techniques in an effort to combine the best of both worlds: the minimal invasiveness of the STING and the same lasting effectiveness as in open surgery. The efficacy and potential advantages or disadvantages of these techniques are still under investigation. The different laparoscopic techniques and available data are presented.
\end{abstract}

Keywords Vesicoureteric reflux · Laparoscopy

\begin{tabular}{|c|c|}
\hline Abbrevi & ations \\
\hline VUR & Vesicoureteric reflux \\
\hline
\end{tabular}

\section{Introduction}

When confronted with the title "What is new in surgical treatment of vesicoureteric reflux?" many readers will automatically think of endoscopic techniques with subureteric injection of bulking agents, also known as STING (Subureteral Teflon INjection). Over the years several substances have been advocated as bulking agents, but the original Teflon is no longer in use. (The most commonly

P. R. H. Callewaert $(\bowtie)$

Department of Urology, University Hospital Maastricht,

PB 5800, 6202 AZ Maastricht,

The Netherlands

e-mail: PCA@urology.azm.nl used substance nowadays is Deflux ${ }^{\mathrm{R}}$, a dextranomer/ hyaluronic acid copolymer.) However, a technique that has been around for a quarter of a century can hardly be considered for a text on surgical novelties. Instead, this review concentrates on the use of laparoscopic techniques in this setting.

\section{General considerations about vesicoureteric reflux}

Vesicoureteric reflux (VUR) remains one of the most frequent conditions in paediatric urology, although the exact prevalence is largely unknown. VUR can be primary, secondary (e.g. to elevated bladder pressures in neurogenic bladders or dysfunctional voiding) and sometimes intermittent in nature, only disclosing itself when infection has possibly induced a degree of insufficiency of the ureterovesical junction. It is generally assumed that VUR predisposes to urinary tract infections and that surgical treatment of reflux and prophylactic antibiotics are equivalent in terms of preventing infections and renal scarring. The relative merit of these interventions in the natural course of these conditions remains to some extent controversial [32].

The importance of voiding dysfunction with detrusor overactivity, underactivity or dysfunctional elimination disorder in the aetiology of VUR should not be underestimated [4, 30] and this has its implications in the treatment offered to these children. Hence bladder training and minimally invasive techniques have acquired a prominent role over the years. Children with VUR and concomitant voiding dysfunction are likely to suffer more breakthrough infections and have lower spontaneous resolution rates and therefore represent a large proportion of the patients undergoing surgical intervention [31]. 
Antimicrobials form the mainstay in the treatment of VUR, in combination with other conservative measures, because VUR will spontaneously disappear in a majority of children and rarely gives rise to serious long-term complications [2]. Increasingly however the exact role of prophylaxis is being questioned as well-designed prospective trials are rare [10]. Nevertheless, a small subgroup of patients does pose problems of break-through infections despite all conservative measures and in fact some of them seem prone to renal scarring leading to hypertension and exceptionally even end stage renal failure [16].

\section{Traditional surgical techniques in the treatment of VUR}

Since the 1950s several surgical techniques have been developed for the correction of VUR. All techniques share the same basic principle of creating an anti-reflux mechanism by increasing the portion of the distal ureter lying in a submucosal tunnel between the detrusor muscle and the bladder mucosa. They offer comparable and very high success rates with few complications [12]. From a purely technical standpoint, these open techniques can basically be divided into two groups. There are those that involve mainly or entirely intravesical ureteral dissection (and hence a need for postoperative bladder drainage) and those that use a purely extravesical approach to the ureter without disconnecting it from the bladder. To the former group belong the techniques of Politano and Leadbetter (1958), Glenn and Anderson (1967), the psoas-hitch technique and the (most widely used) Cohen technique (1975) [6, 11, 13, 25]. In these techniques the ureter is disconnected from the bladder and reimplanted in a new and longer submucosal tunnel from the luminal side of the bladder. In the Cohen technique, a cross-trigonal tunnel is created bringing the ureter to the contralateral side, the other techniques result in a more natural course of the ureter, but are somewhat more prone to complications such as bowel injury or kinking of the ureter. The psoas-hitch technique is generally reserved for more complex situations as in mega-ureters or re-do surgery and is helpful in creating a longer tunnel. The conceptually different extravesical approach was popularized by Lich and Gregoir, reducing postoperative bladder irritation to insignificance, but predisposing to temporary bladder retention when performed bilaterally $[14,21]$.

The more recent and certainly minimally invasive STING technique where bulking agents are injected submucosally has gained wide acceptance. Undoubtedly this is technically a very easy, relatively cheap and patientfriendly treatment modality, tempting many doctors into an increasingly pre-emptive approach to VUR, using it as firstline treatment in cases of (antenatally detected) high-grade reflux even in infants [27]. Success rates, even in low-grade reflux, are clearly lower than in open surgery and a second injection of bulking agent is often necessary [8]. Moreover, prospective randomised trials and long-term results are still not available. The tendency to use this endoscopic technique as an alternative to medical treatment is underscored by the fact that since the Food and Drug Administration (FDA) approval of Deflux ${ }^{\mathrm{R}}$ the total number of procedures for reflux has increased, while open surgery rates have remained stable [20].

All these facts and tendencies mentioned above in turn suggest that, at least for the foreseeable future, there will remain a group of patients in whom STING is deemed-or proves to be-insufficient. Open surgery on the other hand has its drawbacks as well due to its invasiveness. In an ideal world physicians would be able to define very precisely and at the earliest possible point in time which group of patients with VUR is at increased risk for the complication of pyelonephritic scarring and which group is not. This would in turn allow a very tailored approach to each individual child with pre-emptive surgical measures in the group at risk. Failing this knowledge, the next best thing to aim for is to combine the superior results of time-honoured open procedures like a Cohen reimplantation or Lich-Gregoir operation with the much sought after minimal invasiveness of laparoscopy, possibly with the added ultra-precise tissue handling and dexterity of robotic surgery. These considerations are the driving force of the developments described in this text.

\section{Conventional laparoscopic techniques}

Both intra- and extravesical laparoscopic treatments have been described in a great variety of techniques. Most series however remain small and follow-up is very limited. Ehrlich et al. and Janetschek et al. were the first to report in 1994 and 1995 on two and six children undergoing laparoscopic Lich-Gregoir anti-reflux surgery for vesicoureteral reflux [7, 17]. The reflux was successfully corrected without morbidity, requiring only a short hospitalisation. Peri-operative ureteral stents were deemed unnecessary. One mild unilateral stenosis did develop later, requiring temporary stenting. Ehrlich et al. described decreased periand post-operative pain and improved cosmesis by comparison with open surgery. He suggested that this preliminary report deserved further study. Janetschek et al. on the other hand concluded that the Lich-Gregoir anti-reflux procedure was a complicated one because of the difficult suturing and knot-tying, offering no clear advantage over the conventional procedure. Other teams were very reluctant to join in the efforts to develop this approach for several years to come. The choice for a Lich-Gregoir 
technique for the first attempts at correction of VUR can be explained by the fact that, at that time, experience with laparoscopy in cavities other than the abdomen was very limited. Five years later Lakshmanan and Fung reported technical modifications to further minimize invasiveness, basically by downsizing ports and instruments and limiting tissue dissection [19]. A more recent paper by Riquelme et al. again reported excellent outcomes in 15 children, even in cases of bilateral reflux and duplex ureters [28]. There was no postoperative voiding dysfunction. Laparoscopic ureteral reimplantation with extracorporeal tailoring and stenting of megaureters combined with a Lich-Gregoir type of extravesical reimplantation was recently reported by Ansari et al. in three children [1].

Although the Cohen procedure was the more widely used in the treatment of VUR, a laparoscopic version thereof was investigated later than the extravesical laparoscopic techniques. The obvious reason is the anticipated difficulties with port placement and the limitations of the intravesical working space. Different approaches were used by Gill et al. and Yeung et al. [12,33]. Gill et al. combined the use of two suprapubic ports with a transurethral resectoscope for unilateral cases whereas Yeung et al. used three suprapubic ports, more closely copying the open Cohen procedure.

A recent report by Kutikov et al. on either transvesical laparoscopic cross-trigonal ureteral reimplantation in patients with reflux or a Glenn-Anderson reimplantation in patients with a primary obstructing mega-ureter mentions operative success in 25 of 27 patients with VUR and 4 out of 5 patients with mega-ureters, results that are comparable to the ones obtained in open surgery [18]. Complications were postoperative urinary leak in four patients and ureteral stricture at the anastomosis in two. The authors noted that most complications occurred in the younger patients with small bladder capacities.

For completeness two papers on reimplantations in (young) adults can be mentioned. Chung et al. described successful laparoscopic nonrefluxing ureteral reimplantation with a psoas hitch using a submucosal tunnelling technique after submucosal injection of saline under cystoscopy in two adult female patients without postoperative complications [5]. Also in 2006, Puntambekar et al. described laparoscopic extravesical ureteroneocystostomy with psoas hitch in five gynaecologic patients, clearly minimizing the procedural morbidity [26]. Again no intraoperative or postoperative complications occurred.

Gradually more relevant series with larger numbers of patients and longer follow-up are being presented. At the 2007 European Society for Paediatric Urology (ESPU) annual meeting two groups will present their experience in about 80 patients each, with success rates above $90 \%$ (http://www.espu.org).

\section{Robot-assisted techniques}

Over the last 2 years a few authors reported robot-assisted laparoscopic techniques using the $\mathrm{Da} \mathrm{Vinci}^{\mathrm{R}}$ (Intuitive Surgical, Mountain View, CA) system for the treatment of VUR, adding yet another approach to this rapidly expanding field $[3,23,24]$. They made good use of the experience gained with conventional laparoscopy, adding the advantages of robotics: enhanced dexterity of the instruments, absence of tremor and 3-D vision. The generally used term of "robotic surgery" is to some extent actually misleading because it suggests completely autonomous function of the equipment. In reality it works as a master-slave system, merely transferring the movements of the surgeon's hands to the tip of the instruments (Fig. 1). The evolution parallels the one seen in conventional laparoscopy, experience having started with the extravesical approach and later moving to intravesical procedures. The sequence of surgical steps of both techniques will briefly be discussed. As stated, they closely mirror the steps in conventional laparoscopy.

\section{Extravesical technique}

To start, a cystoscopic evaluation of the relevant anatomy is carried out. The camera port is then placed in the umbilicus and the two working ports in each lower abdominal quadrant. A small transverse peritoneal incision is made on the laterodorsal side of the bladder where the ureter is retrieved. The ureter is then buried in a trough between the mucosa and detrusor to create the anti-reflux mechanism (Figs. 2 and 3). The bladder catheter is removed already at the end of the procedure unless a significant perforation needing suturing of the mucosa has been made.

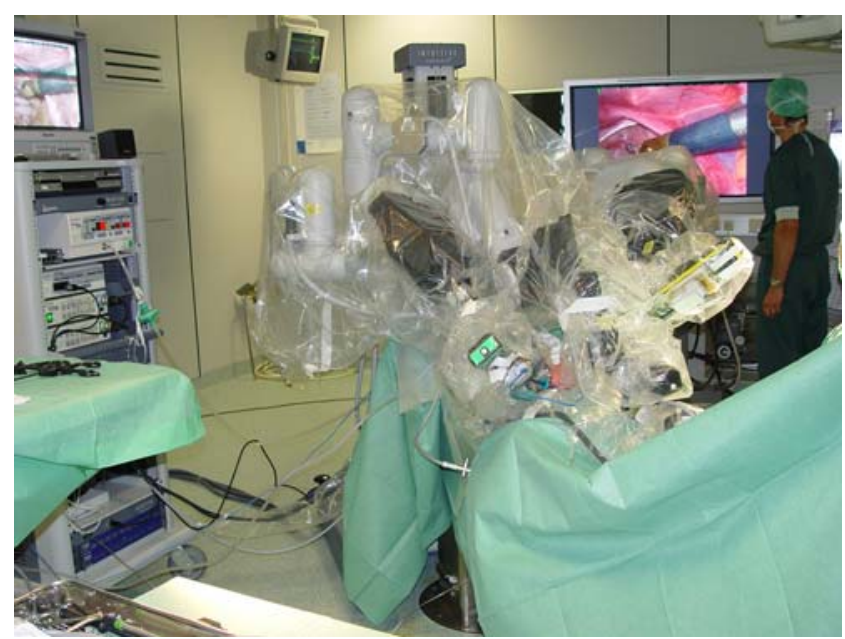

Fig. 1 Outside view once the draped robotic arms are connected to the laparoscopic ports: the child seems completely "embraced" by the machine 


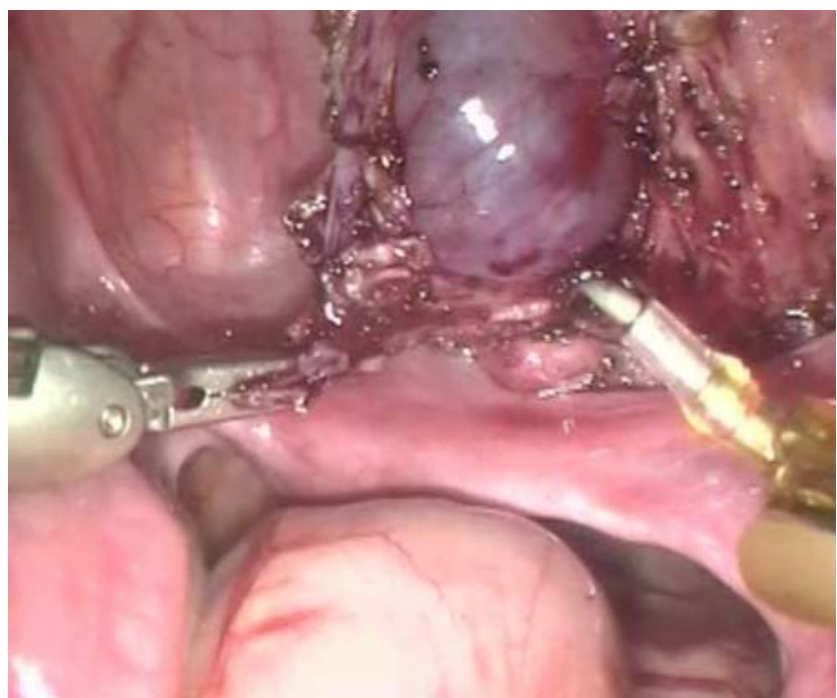

Fig. 2 Extravesical approach: very gently the detrusor muscle is incised and peeled away until the delicate bladder mucosa starts to bulge

In our experience there were no bladder symptoms post surgery in any of the patients. All cases of reflux resolved, but there was one case of "de novo" contralateral low-grade reflux [3]. Later we successfully performed this operation in an adult male patient after a failed subureteral injection (unpublished data). Interestingly, Elmore et al. recently reported on the use of the open Lich-Gregoir technique as salvage in these patients as well [9] and already in 2004 one similar laparoscopic patient was reported by Shu et al. [29]. Both in open and laparoscopic surgery this was a novel approach, meant to avoid the sometimes difficult intravesical dissection due to foreign material after STING. Peters and Borer reported persisting low-grade reflux in 2 of their 24 patients [23].

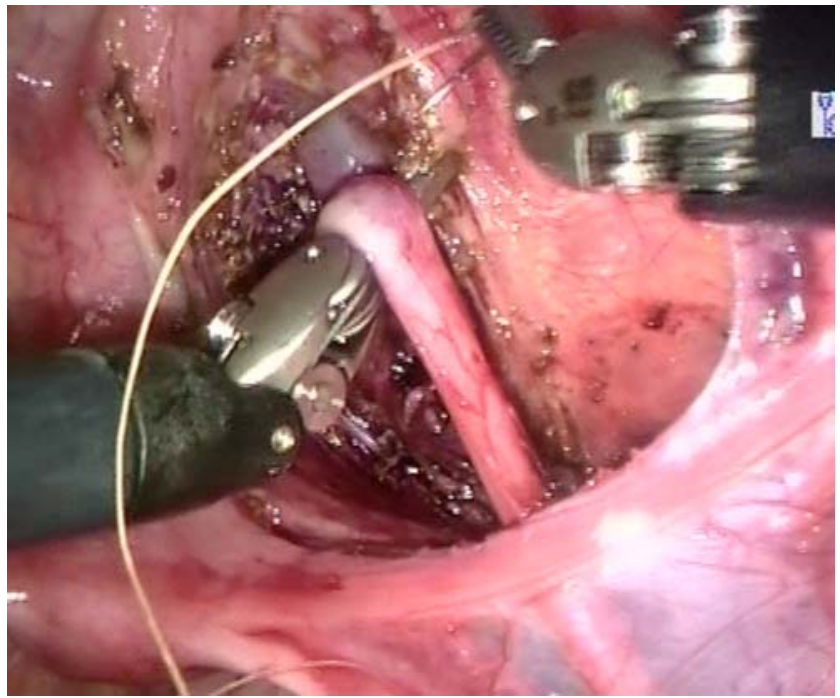

Fig. 3 Extravesical approach: the completely freed ureter is hinged into the trough to create an anti-reflux valve mechanism
Intravesical technique

Olsen was the first to experiment with a Cohen crosstrigonal ureter reimplantation by laparoscopic access to the bladder in a pig model using the Da Vinci ${ }^{\mathrm{R}}$ system [22]. In all pigs the reflux disappeared after the procedure. The advantage of the robotic equipment seemed to be the better access to submucosal tunnelling of the ureter and the intravesical suturing of the anastomosis. Peters and Woo in 2005 and Callewaert in 2006 reported their experience with robot-assisted Cohen procedures in six and three paediatric patients respectively $[3,24]$.

Initial port placement and closure of the incisions at the end of the procedure were the crucial steps, the rest of the procedure being straightforward. Once inside the bladder the mucosa is circumferentially incised around the ostium using the cautery hook. After both ureters are freed, a submucosal tunnel connecting the most proximal part of the two mucosal incisions is created, using forceps and scissors (Fig. 4). Creation of the submucosal tunnel and reimplantation of the ureters is remarkably easy because of the three dimensional visualisation and great dexterity inside the very small volume of a child's bladder. The anatomical detail is such that dissection of the plane between the detrusor and mucosa is achieved with more detail than in open surgery. The bladder catheter is left indwelling for 24 to $48 \mathrm{~h}$.

We had one conversion to open surgery out of three cases in our early experience because of port-related problems in a small child [3]. Kutikov et al. using conventional laparoscopy similarly found that the smaller children were more prone to complications and that these procedures were technically more demanding [18]. Peters and Woo on the other hand reported no conversions in a

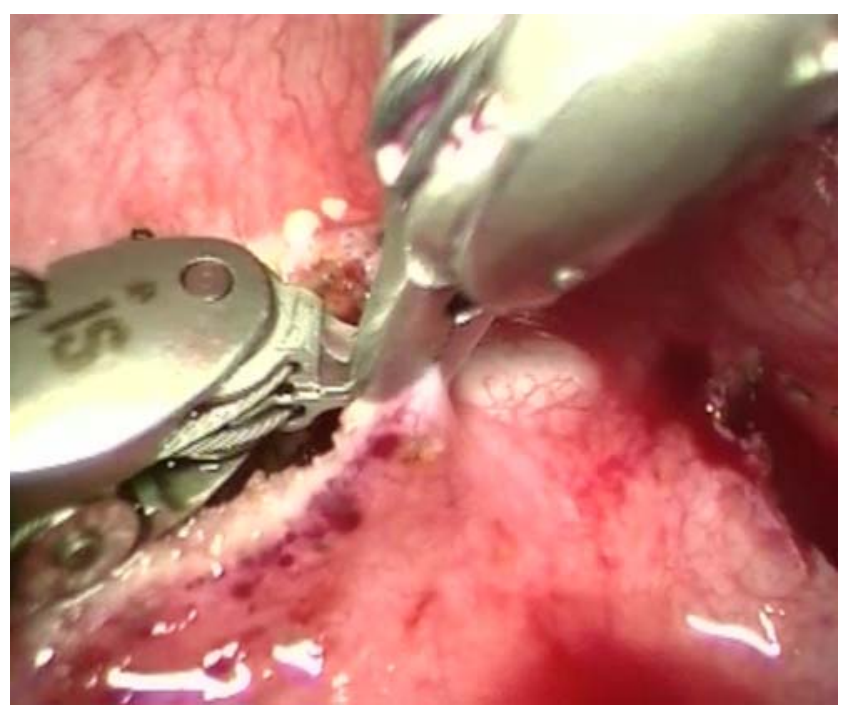

Fig. 4 Intravesical approach: creation of the submucosal tunnel connecting the periureteral incisions. (The jaws of the forceps measure $5 \mathrm{~mm}$ in length) 
series of six children aged between 5 and 15 [24]. They did however have a case of port-site urinary leakage requiring prolonged bladder drainage.

Peters and Callewaert each reported one case of persisting low-grade reflux in their initial experience. Unlike the situation in open surgery, bladder spasms remained completely absent and anticholinergics were unnecessary. This fact is highly suggestive of the minimal invasiveness and limited trauma incurred by the bladder wall.

When comparing the robotically assisted intra- and extravesical operations it is our impression that the LichGregoir technique offers some advantages over the intravesical operation: no need for catheters, no haematuria and easier reproducibility. The drawback is that the abdominal cavity needs to be entered. The abdominal cavity even in smaller children is large enough to allow comfortable movement of the instruments, whereas intravesical operations in this patient group can be technically impossible due in part to the relative bulkiness of the robotic instruments.

\section{Conclusion}

Treatment modalities of reflux are evolving rapidly. Conventional or robot-assisted laparoscopic techniques must be considered a possible future alternative to the more traditional ways of treating this condition. There is no proven superiority at this time and experience is limited to a few centres only and relatively small numbers of patients. It is well established that with open surgery very high success rates can be achieved and that morbidity is relatively low and hospitalisation nowadays can be kept short. The first impressions are that morbidity using laparoscopic techniques is lower still and that there is some cosmetic gain, but it is obvious that the most important issue will be whether the long-term success rates are at least comparable.

Most surgeons agree that robotics certainly add to the precision and ease of the individual surgical steps when compared to conventional laparoscopy, but the financial costs are very high. The intravesical approach using robotics is feasible, but technical difficulties must be taken into account in smaller children. (The same holds true for the conventional laparoscopy.) The extravesical robotic approach clearly seems the more promising, possibly even after failed submucosal injection therapy. Nevertheless we feel that the intravesical approach deserves further pursuing because it may allow surgical correction of other malformations at the level of the bladder neck and ureterovesical junction in a minimally invasive and very precise way.

It would be premature to promote laparoscopy as the golden mean between STING and open surgery for a subgroup of reflux patients at this point, as this would imply diverting a large number of patients to a few centres where either the technical laparoscopic expertise or a robotic system is available. However, we remain convinced that in the (near) future laparoscopy will find its place in the care for these patients.

\section{References}

1. Ansari MS, Mandhani A, Khurana N, Kumar A (2006) Laparoscopic ureteral reimplantation with extracorporeal tailoring for megaureter: a simple technical nuance. J Urol 176(6):26402642

2. Beetz R (2006) May we go on with antibacterial prophylaxis for urinary tract infections? Pediatr Nephrol 21(1):5-13

3. Callewaert P (2006) Intravesical and extravesical robotically assisted laparoscopic antireflux surgery in children: the initial experience. Abstract. J Pediatric Urology 2(2):122-123

4. Chen JJ, Mao W, Homayoon K, Steinhardt GF (2004) A multivariate analysis of dysfunctional elimination syndrome, and its relationships with gender, urinary tract infection and vesicoureteral reflux in children. J Urol 171(5):1907-1910

5. Chung H, Jeong BC, Kim HH (2006) Laparoscopic ureteroneocystostomy with vesicopsoas hitch: nonrefluxing ureteral reimplantation using cystoscopy-assisted submucosal tunneling. J Endourol 20(9):632-638

6. Cohen SJ (1975) Ureterozystoneostomie: eine neue anti-reflux Technik. Aktuelle Urologie 6:1-6

7. Ehrlich RM, Gershman A, Fuchs G (1994) Laparoscopic vesicoureteroplasty in children: initial case reports. Urology 43 (2):255-261

8. Elder JS, Diaz M, Caldamone AA, Cendron M, Greenfield S, Hurwitz R, Kirsch A, Koyle MA, Pope J, Shapiro E (2006) Endoscopic therapy for vesicoureteral reflux: a meta-analysis. I. Reflux resolution and urinary tract infection. J Urol 175(2):716722

9. Elmore JM, Kirsch AJ, Perez-Brayfield MR, Scherz HC, Koyle MA (2006) Salvage extravesical ureteral reimplantation after failed endoscopic surgery for vesicoureteral reflux. J Urol 176 (3): $1158-1160$

10. Garin EH, Olavarria F, Garcia Nieto V, Valenciano B, Campos A, Young L (2006) Clinical significance of primary vesicoureteral reflux and urinary antibiotic prophylaxis after acute pyelonephritis: a multicenter, randomized, controlled study. Pediatrics 117 (3):626-632

11. Gearhart JP, Woolfenden KA (1982) The vesico-psoas hitch as an adjunct to megaureter repair in childhood. J Urol 127(3):505-507

12. Gill IS, Ponsky LE, Desai M, Kay R, Ross JH (2001) Laparoscopic cross-trigonal Cohen ureteroneocystostomy: novel technique. J Urol 166(5):1811-1814

13. Glenn JF, Anderson EE (1967) Distal tunnel ureteral reimplantation. J Urol 97(4):623-626

14. Gregoir W, Vanregemorter G (1964) Le reflux vesico-urétéral congénital. Urol Int 18:122-136

15. Heidenreich A, Ozgur E, Becker T, Haupt G (2004) Surgical management of vesicoureteral reflux in pediatric patients. World $\mathrm{J}$ Urol 22(2):96-106

16. Jacobson SH, Hansson S, Jakobsson B (1999) Vesico-ureteric reflux: occurrence and long-term risks. Acta Paediatr Suppl 88 (431):22-30

17. Janetschek G, Radmayr C, Bartsch G (1995) Laparoscopic ureteral anti-reflux plasty reimplantation. First clinical experience. Ann Urol (Paris) 29(2):101-105 
18. Kutikov A, Guzzo TJ, Canter DJ, Casale P (2006) Initial experience with laparoscopic transvesical ureteral reimplantation at the Children's Hospital of Philadelphia. J Urol 176(5):22222225

19. Lakshmanan Y, Fung LC (2000) Laparoscopic extravesicular ureteral reimplantation for vesicoureteral reflux: recent technical advances. J Endourol 14(7):589-593

20. Lendvay TS, Sorensen M, Cowan CA, Joyner BD, Mitchell MM, Grady RW (2006) The evolution of vesicoureteral reflux management in the era of dextranomer/hyaluronic acid copolymer: a pediatric health information system database study. J Urol $176(4$ Pt 2): 1864-1867

21. Lich R Jr, Howerton LW, Davis LA (1962) Ureteral reflux, its significance and correction. South Med J 55:633-635

22. Olsen LH, Deding D, Yeung CK, Jorgensen TM (2003) Computer assisted laparoscopic pneumovesical ureter reimplantation a.m. Cohen: initial experience in a pig model. APMIS Suppl 109:2325

23. Peters S, Borer J (2005) Robotically assisted laparoscopic antireflux surgery in children. Abstract. J Pediatric Urology 1 (3): 177

24. Peters CA, Woo R (2005) Intravesical robotically assisted bilateral ureteral reimplantation. J Endourol 19(6):618-621

25. Politano VA, Leadbetter WF (1958) An operative technique for the correction of vesicoureteral reflux. J Urol 79(6):932-941
26. Puntambekar S, Palep RJ, Gurjar AM, Sathe RM, Talaulikar AG, Agarwal GA, Kashyap M (2006) Laparoscopic ureteroneocystostomy with psoas hitch. J Minim Invasive Gynecol 13(4):302305

27. Puri P, Pirker M, Mohanan N, Dawrant M, Dass L, Colhoun E (2006) Subureteral dextranomer/hyaluronic acid injection as first line treatment in the management of high grade vesicoureteral reflux. J Urol 176(4 Pt 2):1856-1859

28. Riquelme M, Aranda A, Rodriguez C (2006) Laparoscopic extravesical transperitoneal approach for vesicoureteral reflux. J Laparoendosc Adv Surg Tech A 16(3):312-316

29. Shu T, Cisek LJ Jr, Moore RG (2004) Laparoscopic extravesical reimplantation for postpubertal vesicoureteral reflux. J Endourol 18(5):441-446

30. Sillen U (1999) Bladder dysfunction in children with vesicoureteric reflux. Acta Paediatr Suppl 88(431):40-47

31. Snodgrass W (1998) The impact of treated dysfunctional voiding on the nonsurgical management of vesicoureteral reflux. J Urol 160(5):1823-1825

32. Wheeler DM, Vimalachandra D, Hodson EM, Roy LP, Smith GH, Craig JC (2004) Interventions for primary vesicoureteric reflux. Cochrane Database Syst Rev 3:CD001532

33. Yeung CK, Sihoe JD, Borzi PA (2005) Endoscopic cross-trigonal ureteral reimplantation under carbon dioxide bladder insufflation: a novel technique. J Endourol 19(3):295-299 